\title{
The genome sequence of the common wasp, Vespula vulgaris
}

\section{(Linnaeus, 1758)[version 1; peer review: 3 approved with}

\section{reservations]}

\author{
Liam M. Crowley (iD1,
}

University of Oxford and Wytham Woods Genome Acquisition Lab,

Darwin Tree of Life Barcoding collective,

Wellcome Sanger Institute Tree of Life programme,

Wellcome Sanger Institute Scientific Operations: DNA Pipelines collective, Tree of Life Core Informatics collective, Darwin Tree of Life Consortium

${ }^{1}$ Department of Zoology, University of Oxford, Oxford, OX1 3SZ, UK

V1 First published: 14 Sep 2021, 6:232

https://doi.org/10.12688/wellcomeopenres.17205.1

Latest published: 14 Sep 2021, 6:232

https://doi.org/10.12688/wellcomeopenres.17205.1

\section{Abstract}

We present a genome assembly from an individual female Vespula vulgaris (the common wasp; Arthropoda; Insecta; Hymenoptera; Vespidae). The genome sequence is 188 megabases in span. The majority of the assembly is scaffolded into 25 chromosomal pseudomolecules.

Keywords

Vespula vulgaris, common wasp, genome sequence, chromosomal

This article is included in the Tree of Life gateway.

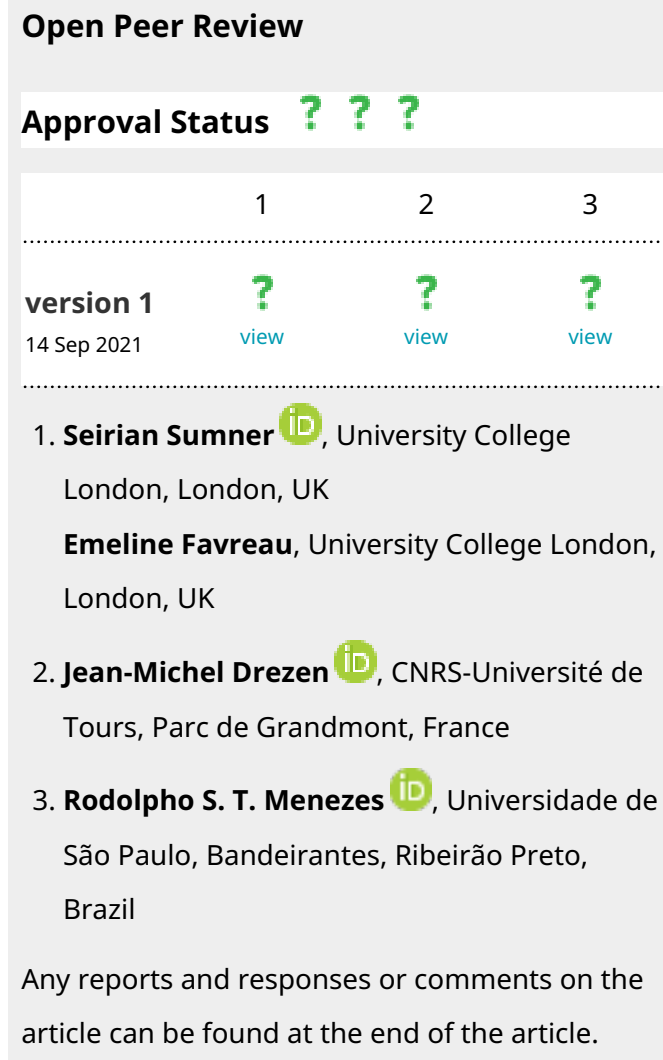


Corresponding author: Darwin Tree of Life Consortium (mark.blaxter@sanger.ac.uk)

Author roles: Crowley LM: Investigation, Resources, Writing - Original Draft Preparation;

Competing interests: No competing interests were disclosed.

Grant information: This work was supported by Wellcome through core funding to the Wellcome Sanger Institute (206194) and the Darwin Tree of Life Discretionary Award (218328).

The funders had no role in study design, data collection and analysis, decision to publish, or preparation of the manuscript.

Copyright: (c) 2021 Crowley LM et al. This is an open access article distributed under the terms of the Creative Commons Attribution License, which permits unrestricted use, distribution, and reproduction in any medium, provided the original work is properly cited.

How to cite this article: Crowley LM, University of Oxford and Wytham Woods Genome Acquisition Lab, Darwin Tree of Life Barcoding collective et al. The genome sequence of the common wasp, Vespula vulgaris (Linnaeus, 1758) [version 1; peer review: 3 approved with reservations] Wellcome Open Research 2021, 6:232 https://doi.org/10.12688/wellcomeopenres.17205.1

First published: 14 Sep 2021, 6:232 https://doi.org/10.12688/wellcomeopenres.17205.1 


\section{Species taxonomy}

Eukaryota; Metazoa; Ecdysozoa; Arthropoda; Hexapoda; Insecta; Pterygota; Neoptera; Endopterygota; Hymenoptera; Apocrita; Aculeata; Vespoidea; Vespidae; Vespinae; Vespula; Vespula vulgaris (Linnaeus, 1758) (NCBI:txid7454).

\section{Introduction}

The Common wasp, Vespula vulgaris, is one of the most widespread species of social wasp in the UK. This species is eusocial, living in colonies with a reproductive queen, sterile workers and reproductive males. Colonies are annual in the UK, typically producing up to around 10,000 workers (Archer, 1981). Nests are commonly constructed underground, particularly within old rodent holes, but can also be frequently found in aerial situations such as roof spaces, sheds, outhouses etc. Nests are constructed out of a paper-like substance produced from macerated wood fibres mixed with saliva. The nest consists of hexagonal cells arranged into combs, covered by a nest envelope. The nest envelope of this species is yellow to brown in colour due to the mix of partially rotted wood used to make the pulp.

Overwintered queens emerge from early March, found a nest around May, and produce the first workers from early June (Archer, 2008). Worker numbers build up throughout the summer, with males and new queens produced around September. Nests usually last until late October, although exceptionally, some may last through the winter to February.

This species is a generalist predator, with workers preying on a wide range of insect and other arthropod species, which are killed, butchered and malaxated before being carried back to the nest to be fed to the developing brood. Adults feed on carbohydrate rich substances including nectar, sap, honeydew and secretions from the larvae. The propensity of adults to visit flowers, particularly shallow blooms, means this species may act as an important pollinator. We note the recent production of a high-quality genome assembly for $V$. vulgaris and two other species of this genus (Harrop et al., 2020), and believe the sequence described here, generated as part of the Darwin Tree of Life project, will further aid understanding of the biology and ecology of the common wasp.

\section{Genome sequence report}

The genome was sequenced from a single female V. vulgaris collected from Wytham Woods, Oxfordshire, UK (latitude 51.774, longitude -1.332). A total of 87 -fold coverage in Pacific Biosciences single-molecule long reads and 190-fold coverage in 10X Genomics read clouds were generated. Primary assembly contigs were scaffolded with chromosome conformation Hi-C data. Manual assembly curation corrected 18 missing/misjoins, reducing the assembly length by $0.001 \%$ and the scaffold number by $34.15 \%$, and increasing the scaffold N50 by $2.74 \%$. The final assembly has a total length of 188 $\mathrm{Mb}$ in 28 sequence scaffolds with a scaffold $\mathrm{N} 50$ of $9 \mathrm{Mb}$ (Table 1). Of the assembly sequence, $99.5 \%$ was assigned to 25 chromosomal-level scaffolds (numbered by sequence length)
Table 1. Genome data for Vespula vulgaris, iyVesVulg1.1.

\begin{tabular}{|c|c|}
\hline \multicolumn{2}{|l|}{ Project accession data } \\
\hline Assembly identifier & iyVesVulg1.1 \\
\hline Species & Vespula vulgaris \\
\hline Specimen & iyVesVulg1 \\
\hline NCBI taxonomy ID & NCBI:txid7454 \\
\hline BioProject & PRJEB43808 \\
\hline BioSample ID & SAMEA7520502 \\
\hline Isolate information & Female, head/thorax \\
\hline \multicolumn{2}{|l|}{ Raw data accessions } \\
\hline PacificBiosciences SEQUEL II & ERR6635597 \\
\hline 10X Genomics Illumina & ERR6054669-ERR6054672 \\
\hline Hi-C Illumina & ERR6054673-ERR6054675 \\
\hline Illumina PolyA RNA-Seq & ERR6286719 \\
\hline \multicolumn{2}{|l|}{ Genome assembly } \\
\hline Assembly accession & GCA_905475345.1 \\
\hline Accession of alternate haplotype & GCA_905404185.1 \\
\hline Span (Mb) & 188 \\
\hline Number of contigs & 50 \\
\hline Contig N50 length (Mb) & 9 \\
\hline Number of scaffolds & 28 \\
\hline Scaffold N50 length (Mb) & 9 \\
\hline Longest scaffold (Mb) & 20 \\
\hline BUSCO* genome score & $\begin{array}{l}\text { C:96.4\%[S:96.1\%,D:0.3\%],F: } \\
\text { 0.8\%,M:2.8\%,n:5991 }\end{array}$ \\
\hline \multicolumn{2}{|c|}{$\begin{array}{l}\text { *BUSCO scores based on the hymenoptera_odb10 BUSCO set using } \\
\text { v5.1.2. C= complete [S= single copy, D=duplicated], F=fragmented, } \\
\mathrm{M}=\text { =missing, n=number of orthologues in comparison. A full set of } \\
\text { BUSCO scores is available at https://blobtoolkit.genomehubs.org/view/ } \\
\text { Vespula\%20vulgaris/dataset/CAJQFS01/busco. }\end{array}$} \\
\hline
\end{tabular}

(Figure 1-Figure 4; Table 2). The assembly has a BUSCO (Simão et al., 2015) v5.1.2 completeness of $96.4 \%$ using the hymenoptera_odb10 reference set. While not fully phased, the assembly deposited is of one haplotype. Contigs corresponding to the second haplotype have also been deposited.

\section{Methods}

A single female $V$. vulgaris was collected from Wytham Woods, Oxfordshire, UK (latitude 51.774, longitude -1.332) by Liam Crowley, University of Oxford, using a net. The sample was snap-frozen using dry ice and stored in a CoolRack.

DNA was extracted from head/thorax tissue of iyVesVulg1 at the Wellcome Sanger Institute (WSI) Scientific Operations 


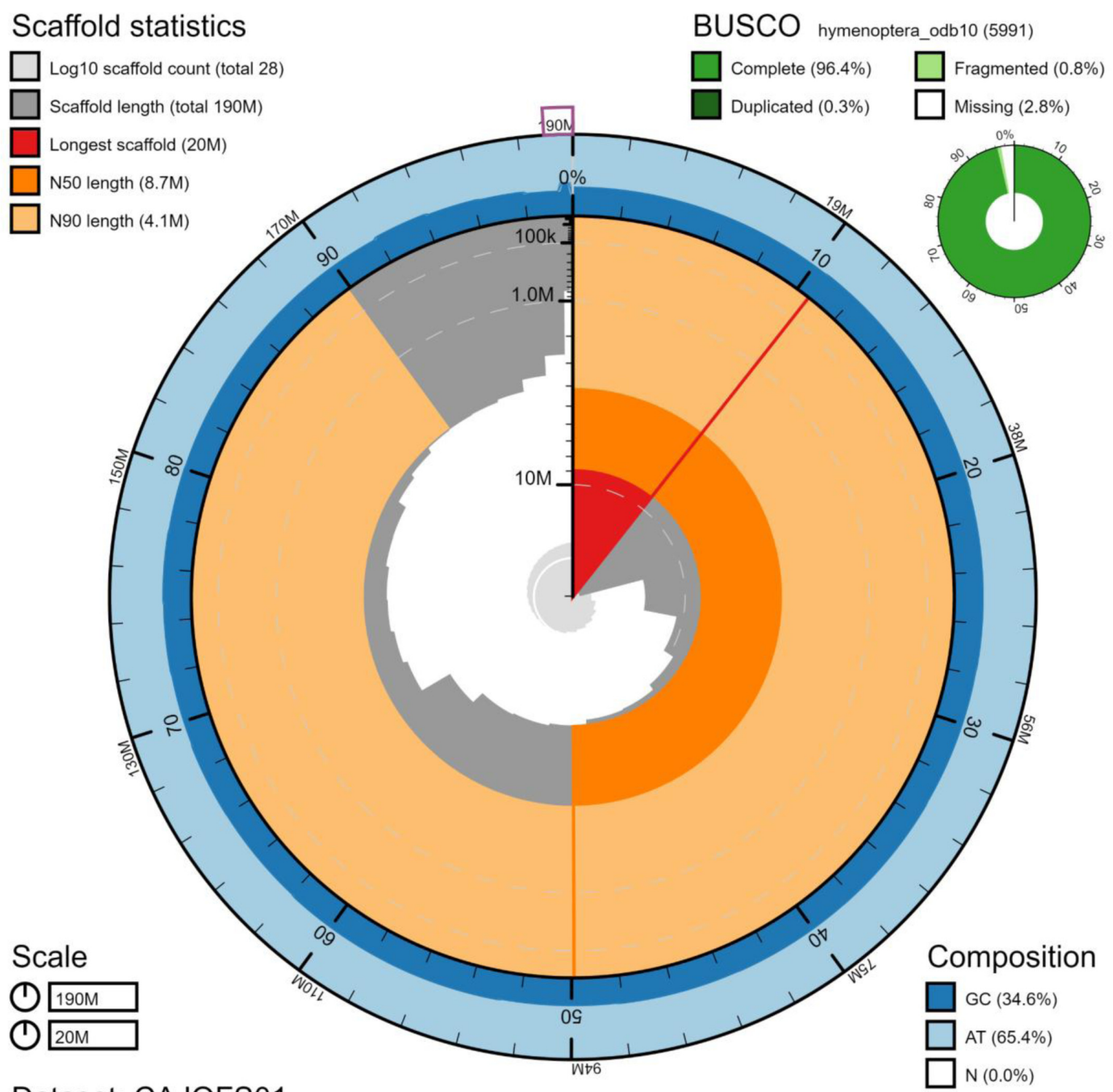

\section{Dataset: CAJQFS01}

Figure 1. Genome assembly of Vespula vulgaris, iyVesVulg1.1: metrics. The BlobToolKit Snailplot shows N50 metrics and BUSCO gene completeness. An interactive version of this figure is available at https://blobtoolkit.genomehubs.org/view/Vespula\%20vulgaris/dataset/ CAJQFS01/snail.

core from the whole organism using the Qiagen MagAttract HMW DNA kit, according to the manufacturer's instructions. RNA (also from the whole organism) was extracted in the Tree of Life Laboratory at the WSI using TRIzol, according to the manufacturer's instructions. RNA was then eluted from the same tissue in $50 \mu$ l RNAse-free water and its concentration RNA assessed using a Nanodrop spectrophotometer and Qubit Fluorometer using the Qubit
RNA Broad-Range (BR) Assay kit. Analysis of the integrity of the RNA was done using Agilent RNA 6000 Pico Kit and Eukaryotic Total RNA assay. A further sample from the same tissue was prepped for $\mathrm{Hi}-\mathrm{C}$ sequencing in the Tree of Life Laboratory using the Arima v2.0 kit.

Pacific Biosciences HiFi circular consensus and 10X Genomics read cloud sequencing libraries were constructed according 


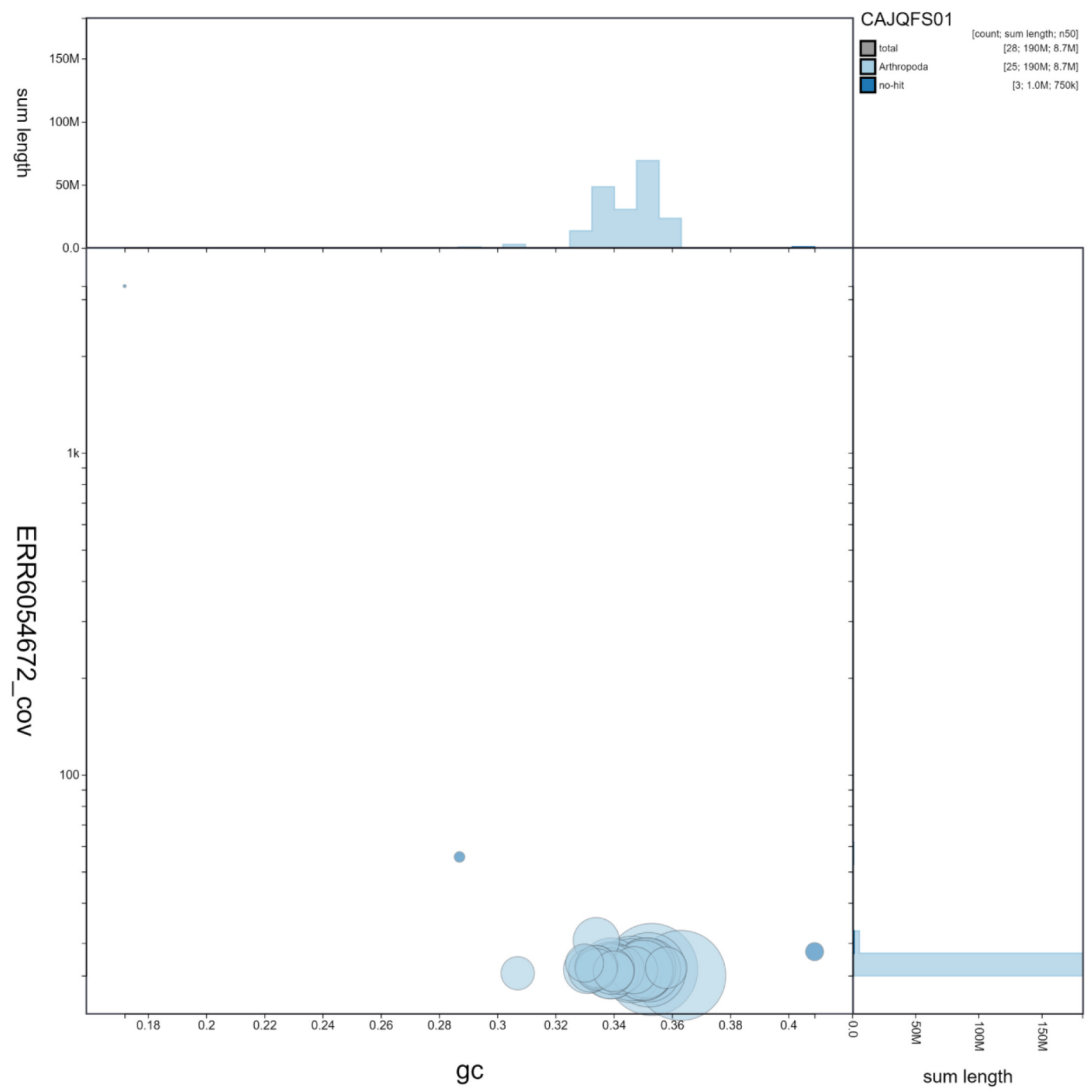

Figure 2. Genome assembly of Vespula vulgaris, iyVesVulg1.1: GC coverage. BlobToolKit GC-coverage plot. Scaffolds are coloured by phylum. Circles are sized in proportion to scaffold length. Histograms show the distribution of scaffold length sum along each axis. An interactive version of this figure is available at https://blobtoolkit.genomehubs.org/view/Vespula\%20vulgaris/dataset/CAJQFS01/blob.

to the manufacturers' instructions. Sequencing was performed by the Scientific Operations core at the Wellcome Sanger Institute on Pacific Biosciences SEQUEL II and Illumina HiSeq X instruments. Hi-C data were sequenced on HiSeq X.

Assembly was carried out with Hifiasm (Cheng et al., 2021). Haplotypic duplication was identified and removed with purge_dups (Guan et al., 2020). One round of polishing was performed by aligning $10 \mathrm{X}$ Genomics read data to the assembly with longranger align, calling variants with freebayes (Garrison \& Marth, 2012). The assembly was then scaffolded with Hi-C data (Rao et al., 2014) was carried out with SALSA2 (Ghurye et al., 2019). The assembly was checked for contamination and corrected using the gEVAL system (Chow et al., 2016) as described previously (Howe et al., 2021). Manual curation was performed using gEVAL, HiGlass 


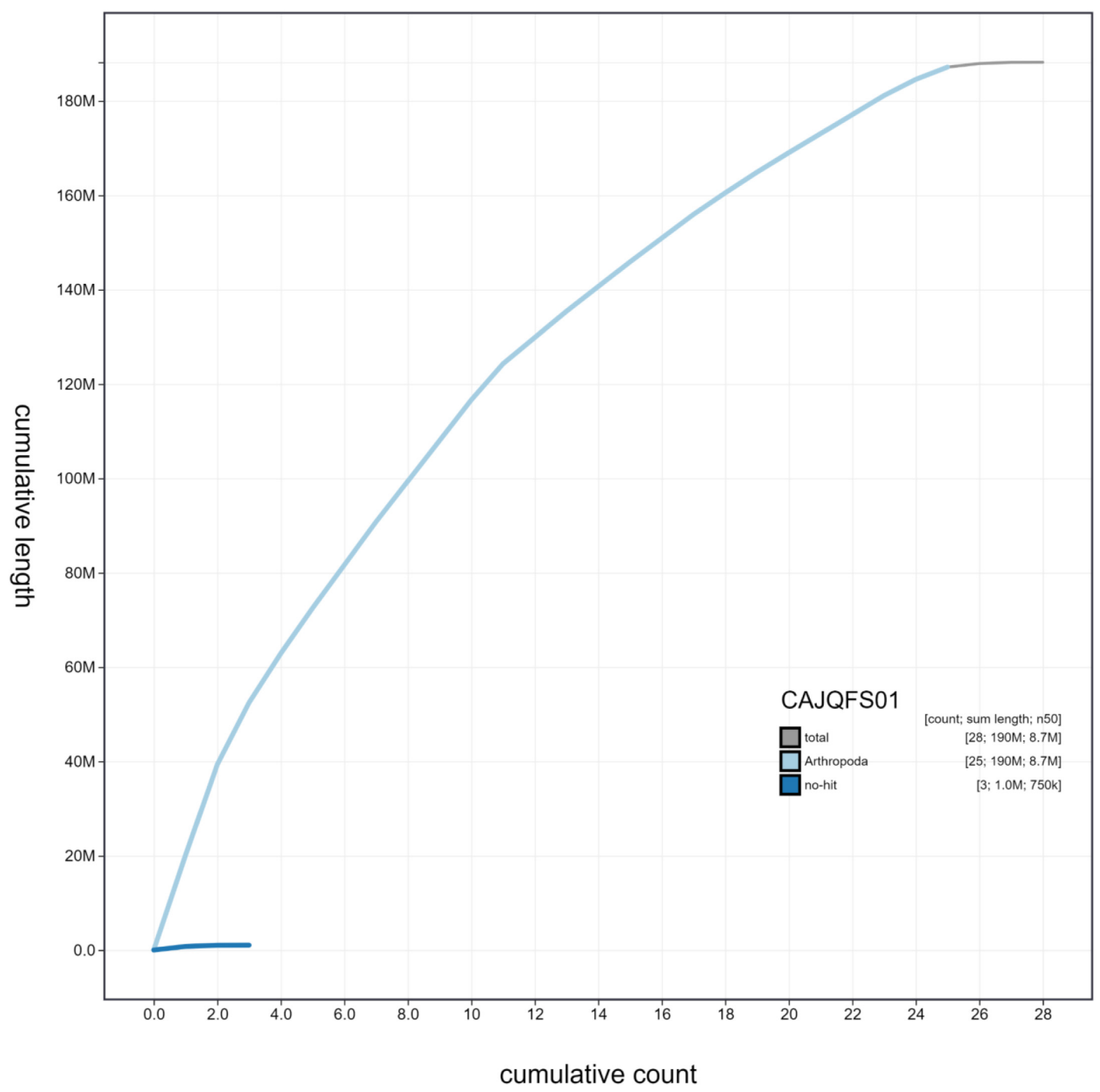

Figure 3. Genome assembly of Vespula vulgaris, iyVesVulg1.1: cumulative sequence. BlobToolKit cumulative sequence plot. The grey line shows cumulative length for all scaffolds. Coloured lines show cumulative lengths of scaffolds assigned to each phylum using the buscogenes taxrule. An interactive version of this figure is available at https://blobtoolkit.genomehubs.org/view/Vespula\%20vulgaris/ dataset/CAJQFS01/cumulative.

(Kerpedjiev et al., 2018) and Pretext. The mitochondrial genome was assembled with MitoHiFi (Uliano-Silva et al., 2021). The genome was analysed and BUSCO scores generated within the BlobToolKit environment (Challis et al., 2020). Table 3 contains a list of all software tool versions used, where appropriate.

The materials that have contributed to this genome note have been supplied by a Darwin Tree of Life Partner. The submission of materials by a Darwin Tree of Life Partner is subject to the Darwin Tree of Life Project Sampling Code of Practice. By agreeing with and signing up to the Sampling Code of Practice, the Darwin Tree of Life Partner agrees they will meet the legal and ethical requirements and standards set out within this document in respect of all samples acquired for, and supplied to, the Darwin Tree of Life Project. Each transfer of samples is further undertaken according to a Research Collaboration Agreement or Material Transfer Agreement entered 


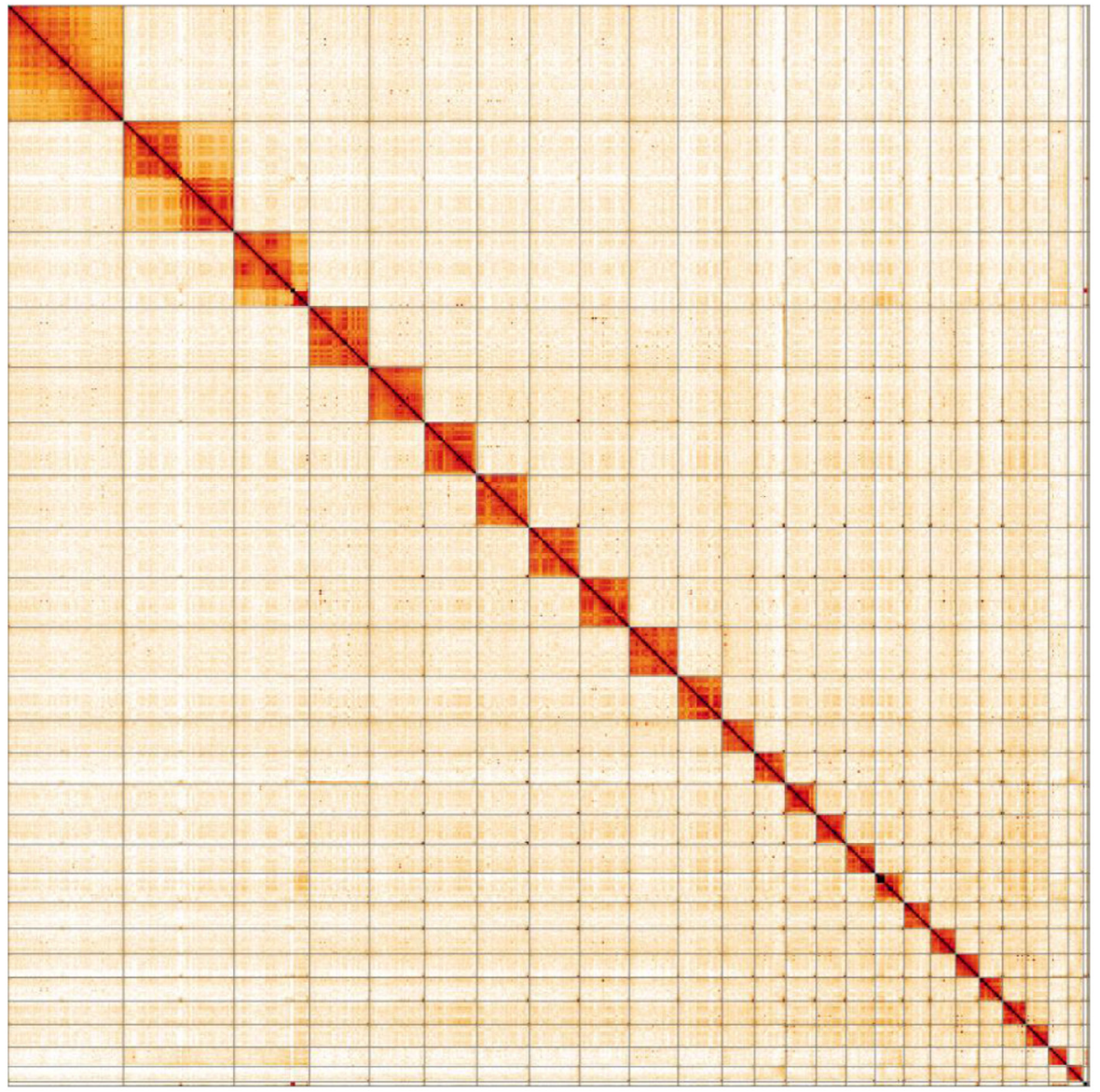

Figure 4. Genome assembly of Vespula vulgaris, iyVesVulg1.1: Hi-C contact map. Hi-C contact map of the iyVesVulg1.1 assembly, visualised in HiGlass.

Table 2. Chromosomal pseudomolecules in the genome assembly of Vespula vulgaris, iyVesVulg1.1.

\begin{tabular}{|c|c|c|c|}
\hline INSDC accession & Chromosome & Size (Mb) & GC\% \\
\hline FR997668.1 & 1 & 20.09 & 35.3 \\
\hline FR997669.1 & 2 & 19.27 & 36.3 \\
\hline FR997670.1 & 3 & 13.10 & 35.2 \\
\hline FR997671.1 & 4 & 10.43 & 34.6 \\
\hline FR997672.1 & 5 & 9.59 & 35.2 \\
\hline FR997673.1 & 6 & 9.17 & 35 \\
\hline FR997674.1 & 7 & 9.11 & 34.5 \\
\hline FR997675.1 & 8 & 8.75 & 33.9 \\
\hline FR997676.1 & 9 & 8.61 & 35.1 \\
\hline FR997677.1 & 10 & 8.52 & 35 \\
\hline FR997678.1 & 11 & 7.65 & 33.9 \\
\hline FR997679.1 & 12 & 5.68 & 34.2 \\
\hline FR997680.1 & 13 & 5.44 & 33.1 \\
\hline
\end{tabular}




\begin{tabular}{|c|c|c|c|}
\hline INSDC accession & Chromosome & Size (Mb) & GC\% \\
\hline FR997681.1 & 14 & 5.26 & 33.9 \\
\hline FR997682.1 & 15 & 5.21 & 34.7 \\
\hline FR997683.1 & 16 & 5.04 & 33.9 \\
\hline FR997684.1 & 17 & 5.02 & 33.4 \\
\hline FR997685.1 & 18 & 4.57 & 33.2 \\
\hline FR997686.1 & 19 & 4.38 & 33.4 \\
\hline FR997687.1 & 20 & 4.13 & 33.4 \\
\hline FR997688.1 & 21 & 4.10 & 33.4 \\
\hline FR997689.1 & 22 & 4.07 & 35.8 \\
\hline FR997690.1 & 23 & 3.95 & 34 \\
\hline FR997691.1 & 24 & 3.41 & 33 \\
\hline FR997692.1 & 25 & 2.61 & 30.7 \\
\hline FR997693.1 & MT & 0.02 & 17.2 \\
\hline- & Unplaced & 1.01 & 37.8 \\
\hline
\end{tabular}

\begin{tabular}{|c|c|c|}
\hline Software tool & Version & Source \\
\hline Hifiasm & 0.12 & Cheng et al., 2021 \\
\hline purge_dups & 1.2 .3 & Guan et al., 2020 \\
\hline SALSA2 & 2.2 & Ghurye et al., 2019 \\
\hline $\begin{array}{l}\text { longranger } \\
\text { align }\end{array}$ & 2.2 .2 & $\begin{array}{l}\text { https://support.10xgenomics.com/genome-exome/ } \\
\text { software/pipelines/latest/advanced/other-pipelines }\end{array}$ \\
\hline freebayes & v1.3.1-17-gaa2ace8 & Garrison \& Marth, 2012 \\
\hline MitoHiFi & 1.0 & https://github.com/marcelauliano/MitoHiFi \\
\hline gEVAL & N/A & Chow et al., 2016 \\
\hline HiGlass & 1.11 .6 & Kerpedjiev et al., 2018 \\
\hline PretextView & $0.1 . x$ & https://github.com/wtsi-hpag/PretextView \\
\hline BlobToolKit & 2.6 .1 & Challis et al., 2020 \\
\hline
\end{tabular}

into by the Darwin Tree of Life Partner, Genome Research Limited (operating as the Wellcome Sanger Institute), and in some circumstances other Darwin Tree of Life collaborators.

\section{Data availability}

European Nucleotide Archive: Vespula vulgaris (common wasp). Accession number PRJEB43808; https://identifiers.org/ena. embl:PRJEB43808.
The genome sequence is released openly for reuse. The $V$. vulgaris genome sequencing initiative is part of the Darwin Tree of Life (DToL) project. All raw sequence data and the assembly have been deposited in INSDC databases. The genome will be annotated using the RNA-Seq data and presented through the Ensembl pipeline at the European Bioinformatics Institute. Raw data and assembly accession identifiers are reported in Table 1. 
Acknowledgements

Members of the University of Oxford and Wytham Woods Genome Acquisition Lab are listed here: https://doi.org/10.5281/ zenodo.4789929.

Members of the Darwin Tree of Life Barcoding collective are listed here: https://doi.org/10.5281/zenodo.4893704.

Members of the Wellcome Sanger Institute Tree of Life programme collective are listed here: https://doi.org/10.5281/ zenodo.5377053.
Members of Wellcome Sanger Institute Scientific Operations: DNA Pipelines collective are listed here: https://doi.org/10.5281/ zenodo.4790456.

Members of the Tree of Life Core Informatics collective are listed here: https://doi.org/10.5281/zenodo.5013542.

Members of the Darwin Tree of Life Consortium are listed here: https://doi.org/10.5281/zenodo.4783559.
Archer ME: A Simulation Model for the Colonial Development of Paravespula Vulgaris (Linnaeus) and Dolichovespula Sylvestris (Scopoli) (Hymenoptera: Vespidae). Melanderia. 1981; 36:1. Reference Source

Archer ME: Taxonomy, Distribution and Nesting Biology of Species of the Genus Paravespula or the Vespula Vulgaris Species Group (Hymenoptera, Vespidae). Entomologist's Monthly Magazine (United Kingdom). 2008; 144: 5-29. Challis R, Richards E, Rajan J, et al.: BlobToolKit - Interactive Quality Assessment of Genome Assemblies. G3 (Bethesda). 2020; 10(4): 1361-74. PubMed Abstract | Publisher Full Text | Free Full Text

Cheng $\mathrm{H}$, Concepcion GT, Feng X, et al.: Haplotype-Resolved de Novo Assembly Using Phased Assembly Graphs with Hifiasm. Nat Methods. 2021 18(2): 170-75.

PubMed Abstract | Publisher Full Text | Free Full Text

Chow W, Brugger K, Caccamo M, et al.: gEVAL - a Web-Based Browser for Evaluating Genome Assemblies. Bioinformatics. 2016; 32(16): 2508-10. PubMed Abstract | Publisher Full Text | Free Full Text

Garrison E, Marth G: Haplotype-Based Variant Detection from Short-Read Sequencing. arXiv: 1207.3907. 2012.

Reference Source

Ghurye J, Rhie A, Walenz BP, et al.: Integrating Hi-C Links with Assembly Graphs for Chromosome-Scale Assembly. PLoS Comput Biol. 2019; 15(8): e1007273.

PubMed Abstract | Publisher Full Text | Free Full Text

Guan D, McCarthy SA, Wood J, et al.: Identifying and Removing Haplotypic
Duplication in Primary Genome Assemblies. Bioinformatics. 2020; 36(9): 2896-98.

PubMed Abstract | Publisher Full Text | Free Full Text

Harrop TWR, Guhlin J, McLaughlin GM, et al.: High-Quality Assemblies for Three Invasive Social Wasps from the Vespula Genus. G3 (Bethesda). 2020; 10(10): 3479-88.

PubMed Abstract | Publisher Full Text | Free Full Text

Howe K, Chow W, Collins J, et al.: Significantly Improving the Quality of Genome Assemblies through Curation. GigaScience. 2021; 10(1): giaa153. PubMed Abstract | Publisher Full Text | Free Full Text

Kerpedjiev P, Abdennur N, Lekschas F, et al.: HiGlass: Web-Based Visua Exploration and Analysis of Genome Interaction Maps. Genome Biol. 2018; 19(1): 125.

PubMed Abstract | Publisher Full Text | Free Full Text

Rao SS, Huntley MH, Durand NC, et al:: A 3D Map of the Human Genome at Kilobase Resolution Reveals Principles of Chromatin Looping. Cell. 2014; 159(7): 1665-80.

PubMed Abstract | Publisher Full Text | Free Full Text

Simão FA, Waterhouse RM, Ioannidis P, et al: BUSCO: Assessing Genome Assembly and Annotation Completeness with Single-Copy Orthologs. Bioinformatics. 2015; 31(19): 3210-12.

PubMed Abstract | Publisher Full Tex

Uliano-Silva M, Nunes JGF, Krasheninnikova K, et al.: marcelauliano/MitoHiFi: mitohifi v2.0. 2021

Publisher Full Text 


\section{Open Peer Review}

\section{Current Peer Review Status: ? ? ?}

\section{Version 1}

Reviewer Report 06 January 2022

https://doi.org/10.21956/wellcomeopenres.19012.r47289

(c) 2022 Menezes R. This is an open access peer review report distributed under the terms of the Creative Commons Attribution License, which permits unrestricted use, distribution, and reproduction in any medium, provided the original work is properly cited.

\section{Rodolpho S. T. Menezes}

Department of Biology, Faculty of Philosophy, Universidade de São Paulo, Bandeirantes, Ribeirão Preto, Sao Paulo, 14040-901, Brazil

The manuscript by Crowley et al. present a genome assembly from a female Vespula vulgaris. The genome sequence is 188 megabases in span, a very similar result found by Harrop et al. (2020) ${ }^{1}$. Overall the manuscript presents an advance in the genomic field of social wasps. The methodology is thorough and explained and the figures are well constructed. However, I think the authors should compare they results with the assembled genomes from the three Vespula species, specially Vespula vulgaris as reported by Harrop et al., (2020) ${ }^{1}$. Thus, considering a comparative context will better justify the genome sequence of two individuals of the same species.

\section{References}

1. Harrop T, Guhlin J, McLaughlin G, Permina E, et al.: High-Quality Assemblies for Three Invasive Social Wasps from the Vespula Genus. G3: Genes, Genomes, Genetics. 2020; 10 (10): 3479-3488 Publisher Full Text

Is the rationale for creating the dataset(s) clearly described? Partly

Are the protocols appropriate and is the work technically sound? Yes

\section{Are sufficient details of methods and materials provided to allow replication by others?} Yes

Are the datasets clearly presented in a useable and accessible format? Yes

Competing Interests: No competing interests were disclosed. 
Reviewer Expertise: Cytogenetics, phylogenomics, and phylogeography.

\section{I confirm that I have read this submission and believe that I have an appropriate level of expertise to confirm that it is of an acceptable scientific standard, however I have significant reservations, as outlined above.}

Reviewer Report 21 December 2021

https://doi.org/10.21956/wellcomeopenres.19012.r47264

(C) 2021 Drezen J. This is an open access peer review report distributed under the terms of the Creative Commons Attribution License, which permits unrestricted use, distribution, and reproduction in any medium, provided the original work is properly cited.

\section{Jean-Michel Drezen \\ Insect Biology Research Institute, CNRS-Université de Tours, Parc de Grandmont, Tours, 37200, France}

The genome report of the common wasp Vespula vulgaris begins with an introduction describing the natural history of the wasp, followed by technical aspects of genome sequencing performed to obtain high-quality genome chromosome scale assembly. The introduction is well written and will be of interest for a broad audience, whereas the detailed protocol and figures will be understood only by specialists in genomics, which is acceptable if it is a clear choice to do so for these short papers, if not more explanations are needed in the figure legends and methods part. The genome obtained is of high quality and the most important point of the protocol are described. However, some points could be improved.

In the introduction the exclusive reference to the UK fauna seems a little surprising since the original geographical distribution of this species is Eurasia from UK to China, it should be stated somewhere, the wasp is not an endemic species of UK! It is also noteworthy that this species has also been recorded as an invasive pest in different parts of the world such as South America, Australia and New Zealand and may have a strong impact on local fauna because of its proliferation in these areas of invasion (Lester and Beggs, $2019^{1}$ ).

I understand that the genome note should be very concise but this accurate natural history could have been written as well centuries ago and some references to current research themes on wasps would be of interest for example the study of venom components, of high recombination rates in Hymenoptera, the evolution of sociality, behavior (Herrera et al. 20202; Sirviö et al., 2013³; etc...)

Few genomes from Hymenoptera have been obtained at the chromosomal scale and this an important achievement, however this is the second one for this species, as appropriately noted. A comparison between the two genomes obtained, at least at first glance, would be of interest, in particular the repetitive sequences (including transposable elements) recovered in obtained genomes may vary depending on the technical approach used. 
Figure 1: The meaning of colored circle in the middle of the figure using most of the space available is difficult to understand. It provides almost no additional information since the important numbers are already indicated in "scaffold statistics", "scale", "busco" and "composition". I would reorganize the figure to give more space to the important data by reducing the size of this circle, it could be the size of the green one (Busco).

Figure 2: Some explanations on the principle of blobtool (what distribution of GC content and coverage might tell?) and what is shown on the $y$ axis is required for non-specialists to understand something. As it stands only researcher deeply involved in genomics can make something of this figure. At what might correspond the circles having different GC proportion (lower with higher coverage and higher with similar coverage)? Some words are required in the legend to help nonspecialists.

Figure 4: Same remark - it may worth writing that each square corresponds to a chromosome.

In the Methods section it is not clear which tissue was used for RNA extraction is it head/thorax (indicated in the first sentence) or the whole body, as indicated in the second sentence?

It would be useful for the reader to know how many genes are included in the hymenoptera_odb10 reference set since the result of Busco depends on the hymenoptera orthologous genes which is is not stable yet and might decrease as more genomes get sequenced, in principle all highly conserved hymenopteran genes should be recovered in a chromosomal scale genome. For example, testing a Busco set of 1658 genes we recovered more than 99\% orthologues in the parasitoid wasp C. congregata genome (Gauthier et al., 2021 ${ }^{4}$ (my paper)). Either some of the genes in the Busco set used are not highly conserved and should not be included in the set used or the genome is not complete regarding gene content.

It is a general practice to check for contamination but insect genomes may contain insertions of virus sequences (Gasmi et al., $2015^{5}$ (my paper)) or of bacterial genomes (Wolbachia, Klasson et al., $2014^{6}$ ) that are deleted after checking for "alien sequences", although they are actual part of the genome. How the approach used here (Geval) is dealing with this problem?

\section{References}

1. Lester P, Beggs J: Invasion Success and Management Strategies for SocialVespula Wasps. Annual Review of Entomology. 2019; 64 (1): 51-71 Publisher Full Text

2. Herrera C, Leza M, Martínez-López E: Diversity of compounds in Vespa spp. venom and the epidemiology of its sting: a global appraisal.Arch Toxicol. 94 (11): 3609-3627 PubMed Abstract | Publisher Full Text

3. Sirviö A, Johnston JS, Wenseleers T, Pamilo P: A high recombination rate in eusocial

Hymenoptera: evidence from the common wasp Vespula vulgaris.BMC Genet. 2011; 12: 95 PubMed Abstract | Publisher Full Text

4. Gauthier J, Boulain H, van Vugt J, Baudry L, et al.: Chromosomal scale assembly of parasitic wasp genome reveals symbiotic virus colonization. Communications Biology. 2021; 4 (1). Publisher Full Text

5. Gasmi L, Boulain H, Gauthier J, Hua-Van A, et al.: Recurrent Domestication by Lepidoptera of Genes from Their Parasites Mediated by Bracoviruses.PLoS Genet. 2015; 11 (9): e1005470 PubMed Abstract | Publisher Full Text

6. Klasson L, Kumar N, Bromley R, Sieber K, et al.: Extensive duplication of the Wolbachia DNA in 
chromosome four of Drosophila ananassae.BMC Genomics. 2014; 15: 1097 PubMed Abstract |

Publisher Full Text

Is the rationale for creating the dataset(s) clearly described?

Yes

Are the protocols appropriate and is the work technically sound?

Yes

Are sufficient details of methods and materials provided to allow replication by others? No

Are the datasets clearly presented in a useable and accessible format?

Partly

Competing Interests: No competing interests were disclosed.

Reviewer Expertise: Insect viruses, polydnaviruses, parastoid wasps genomics, evolution

I confirm that I have read this submission and believe that I have an appropriate level of expertise to confirm that it is of an acceptable scientific standard, however I have significant reservations, as outlined above.

Reviewer Report 05 November 2021

https://doi.org/10.21956/wellcomeopenres.19012.r45893

(C) 2021 Sumner S et al. This is an open access peer review report distributed under the terms of the Creative Commons Attribution License, which permits unrestricted use, distribution, and reproduction in any medium, provided the original work is properly cited.

\section{Seirian Sumner}

Centre for Biodiversity and Environment Research, University College London, London, UK

\section{Emeline Favreau}

Centre for Biodiversity and Environment Research, University College London, London, UK

\section{Summary}

The genome report of the common wasp Vespula vulgaris includes a short, to the point natural history description (level of sociality, life cycle, ecology), followed by the protocols (one wasp, longread sequencing, $\mathrm{Hi}-\mathrm{C}$ ) to obtain the high-quality genome assembly. We commend the efforts to produce long-reads and a near-chromosomal-level assembly from a single female wasp Vespula vulgaris. As a result of state-of-the-art sequencing methods, the assembly contains an expected high amount of complete protein-coding genes (BUSCO score) and 28 scaffolds within the range of chromosome count previously found in Vespula species, including this species (Harrop et al 2020). The readily availability of the high-quality assembly will help the wasp research community to produce more informed comparative analyses. At the time of this publication, there are few 
wasp genomes assembled at chromosomal level, thus the rationale for assembling this genome is well described.

\section{Comments:}

1. A reference that details the ecosystem services of this wasp as pollinator and predator/regulator of insect populations could be included e.g. Brock et al. (2021) ${ }^{1}$.

2. The authors note, correctly, that this is not the first high quality genome sequence for this species, but it is the first for a UK representative. There is little/no comparison of this genome with the previously published one; this may be beyond the scope of a short genome report like this.

3. DNA for both PacBio and Hi-C sequencing, as well as RNA sequencing was all obtained from the sample single individual. Sequencing DNA from a single diploid individual is preferable as it reduces heterozygosity; however, a male would have been even better, as hymenopteran males are haploid. Was the female a queen or worker? This should be stated as it could be useful for future population genetics studies.

4. Information on the amount of sequencing conducted (e.g. number of reads) appears to be missing.

5. RNA appears to have been obtained from a whole body, although this is not entirely clear; e.g. it could be that the single female was cut in half and half used for DNA and half for RNA. This wouldn't matter for the DNA sequencing but it could affect the repertoire of genes represented in the RNA sample, e.g. if some organs were present in one half but not the other. Future work might consider adding brood types and males to the RNA pool to improve annotations.

6. To make the article reproducible, the report would need to include a link to the bioinformatics steps taken and code used. Table 3 list the software tools used, but there is no description of specific options used for each step, for instance whether hifiasm was used with the Hi-C integration or freebayes options. Here is a Wellcome Open research example on Github: https://github.com/SimonAB/Gonzalez-Jimenez_MIRS

7. In the Methods, the mitochondrial method is mentioned. Is it available? If so, where?

8. Figure 1: the scales of the circle are difficult to understand: what is the visual difference between $190 \mathrm{M}$ and and $20 \mathrm{M}$ ? Also, what is the purple rectangle on $190 \mathrm{M}$ ?

9. Figure 2: The legend font size is quite small to read.

10. Figure 4: There are several dots on the Hi-C contact map that could be indicative of misassembly or TAD loci. Have you investigated it?

11. Typo: Remove 2 nd 'was' from this sentence: The assembly was then scaffolded with Hi-C data (Rao et al., 2014) was carried out with SALSA2 (Ghurye et al., 2019). 


\section{References}

1. Brock RE, Cini A, Sumner S: Ecosystem services provided by aculeate wasps.Biol Rev Camb Philos Soc. 96 (4): 1645-1675 PubMed Abstract | Publisher Full Text

Is the rationale for creating the dataset(s) clearly described?

Yes

Are the protocols appropriate and is the work technically sound?

Yes

Are sufficient details of methods and materials provided to allow replication by others? Partly

Are the datasets clearly presented in a useable and accessible format?

Yes

Competing Interests: No competing interests were disclosed.

Reviewer Expertise: Social wasps; social evolution \& behaviour; sociogenomics

We confirm that we have read this submission and believe that we have an appropriate level of expertise to confirm that it is of an acceptable scientific standard, however we have significant reservations, as outlined above. 